\title{
Diversité, structure et régénération de la végétation ligneuse de la Station Sahélienne Expérimentale de Toukounous, Niger
}

\author{
Saidou OUSSEINA ${ }^{1 *}$, Riccardo FORTINA ${ }^{2}$, Hamani MARICHATOU ${ }^{3}$ et \\ Alhassan YENIKOYE ${ }^{4}$ \\ ${ }^{\text {I} F a c u l t e ́ ~ d ' A g r o n o m i e, ~ U n i v e r s i t e ́ ~ A b d o u ~ M o u m o u n i ~ d e ~ N i a m e y, ~ B P ~: ~ 10960, ~ N i a m e y, ~ N i g e r . ~}$ \\ ${ }^{2}$ Dipartimento di Scienze Zootecniche, Università di Torino, Via L. da Vinci 44, 10095 Grugliasco, Italie. \\ ${ }^{3}$ Faculté d'Agronomie, Université Abdou Moumouni de Niamey, BP : 10960, Niamey, Niger. \\ ${ }^{4}$ Faculté d'Agronomie, Université Abdou Moumouni de Niamey, BP : 10960, Niamey, Niger. \\ *Auteur correspondant, E-mail : ousseysk@refer.ne; ousseys@yahoo.fr ; Tél. +27964081 13/90 381874
}

\section{RESUME}

A la Station Sahélienne Expérimentale de Toukounous, le bétail est nourri essentiellement sur pâturage naturel. En saison sèche, l'herbe devient rare et subsiste à l'état de paille. Les animaux ont recours aux ligneux pour combler le déficit alimentaire. L'état de la végétation ligneuse a été établi à travers la détermination de la diversité, la structure et la régénération des espèces ligneuses par la méthode des transects. L'inventaire floristique a permis de recenser 15 espèces réparties entre 11 genres et 10 familles. La famille des Capparaceae et celle des Leguminosae-Mimosoïdeae sont les plus représentées. Le peuplement est une formation arbustive à Maerua crassifolia avec comme espèces compagnes Balanites aegyptiaca et Acacia raddiana. La densité moyenne est de 172 pieds à l'hectare. La distribution par classes de diamètre montre une prédominance d'individus de petit diamètre. La majorité des ligneux a une hauteur inférieure à 2 mètres $(46,4 \%)$. Le degré de couverture de la strate ligneuse, estimé à $12,67 \%$ est globalement faible. Le taux de renouvellement varie selon l'espèce. Maerua crassifolia (47,9\%), Balanites aegyptiaca (17,4\%) et Acacia raddiana $(14,1 \%)$ ont les plus forts peuplements juvéniles. L'effectif de Maerua crassifolia est en baisse. La structure du peuplement fait état d'un milieu perturbé. La protection des jeunes plants devrait permettre de diversifier les sources d'aliments pour le bétail en période de soudure.

(C) 2015 International Formulae Group. All rights reserved.

Mots clés : Peuplement ligneux, parcours, caractéristiques structurales, Sahel, Niger.

\section{INTRODUCTION}

$\mathrm{Au}$ Sahel, les écosystèmes sont caractérisés par une strate herbacée continue parsemée de plantes ligneuses. Dans cette zone, les variations climatiques actuelles et passées enregistrées ces dernières décennies ont entraîné une série de sécheresses dont les plus importantes sont celles des années 19691973 et 1983-1985 (Le Barbé et Lebel, 1997;
Ganaba et al,.2005; Ali et al., 2008; Ali et Lebel, 2009). La végétation sahélienne subit une forte dégradation consécutive à la baisse constante des précipitations enregistrées (Ganaba et Guinko, 1995). Plusieurs auteurs ont rapporté un déplacement des isohyètes vers le sud (Ozer et Erpicum, 1995; Ciofolo, 1995). Ces perturbations climatiques aggravées par la dégradation des sols, 
devenues fréquentes et intenses compromettant ainsi les productions végétale et animale, ont entraîné un déséquilibre entre le disponible fourrager et les effectifs du bétail (Cornet et al., 2002 ; Lhoste, 2007). La forte pression exercée sur les ressources fourragères, accentuée par la péjoration du climat, peut conduire à la réduction voire la disparition de certaines espèces végétales (Grouzis, 1995). Elle peut aussi favoriser le développement des espèces résistantes ou l'émergence d'espèces mieux adaptées aux nouvelles conditions du milieu (Arbonnier, 2000). La dégradation des écosystèmes représente une des plus importantes causes de réduction de la biodiversité dans le monde (FAO, 2007).

Face à la rigueur climatique et à la pression sur les ressources disponibles, la Station Sahélienne Expérimentale de Toukounous a été créée, avec comme objectifs, la sélection, la standardisation, la multiplication, la diffusion en milieu paysan de la race bovine sahélienne (l'azawak) et la formation des jeunes éleveurs traditionnels.

Le système d'élevage pratiqué à la Station de Toukounous est de type extensif, essentiellement basé sur l'exploitation des ressources naturelles (fourrages herbacé et ligneux). Pendant la saison pluvieuse, l'herbe est abondante et assure l'alimentation du bétail. En début de la saison sèche, au moment où les herbacées entament leur dessèchement, les ligneux sont toujours en pleine feuillaison (Fournier, 1991). Lorsque la saison sèche avance, le pâturage herbacé devient rare et subsiste à l'état de paille de faible valeur nutritive. La végétation ligneuse joue alors un rôle très important dans l'alimentation du bétail et assure l'essentiel des apports en azote, vitamines et éléments minéraux pendant la saison sèche. Cependant, très peu d'études ont été conduites sur la végétation ligneuse de la Station (Douma et al., 2007 ; Diatta, 2008).

Aussi, dans les conditions d'élevage telles que celles de la station, où il n'est pas rare de rencontrer des animaux flairant des plages dénudées à la recherche des brins de paille pendant la saison sèche, il est nécessaire de déterminer l'état des ressources fourragères ligneuses pour cerner davantage la problématique de l'alimentation du bétail de la Station de Toukounous. Ce qui permettra d'avoir des bases pour une gestion durable des ressources fourragères de la Station.

L'objectif de cette étude est de déterminer la diversité, la structure et la régénération potentielle du peuplement ligneux de la Station afin de dégager des conclusions permettant de mieux gérer ces ressources.

\section{MATÉRIEL ET MÉTHODES \\ Description de la zone d'étude}

L'étude s'est déroulée à la Station Sahélienne Expérimentale de Toukounous (SSET) au Niger. Elle est située dans la vallée du Dallol Bosso à $20 \mathrm{~km}$ au nord de Filingué (région de Tillabéri) et à $200 \mathrm{~km}$ au nord-est de Niamey à $14^{\circ} 31^{\prime \prime}$ de latitude Nord et $3^{\circ} 18^{\prime \prime}$ de longitude Ouest. Le climat est tropical semi-aride, de type sahélien, caractérisé par une courte saison pluvieuse allant de juillet à septembre et une longue saison sèche (9 mois). La température moyenne journalière varie entre $20{ }^{\circ} \mathrm{C}$ et $45{ }^{\circ} \mathrm{C}$ et le taux d'humidité relative de l'air peut atteindre $70 \%$ en saison pluvieuse puis descendre à $30 \%$, voire $10 \%$ en saison sèche chaude (Diatta et al., 2004). La pluviosité moyenne interannuelle est de 351,2 mm (Ousseina, 2012). Les sols sont de type sableux $(80 \%)$ avec un modelé légèrement ondulé et entrecoupé par des dépressions argileuses inondées et impraticables en période pluvieuse (Achard et Chanono, 1995). La végétation est à dominance de graminées annuelles telles que Schoenefeldia gracilis Kunth., Aristida mutabilis Trin. et Rupr., Dactyloctenium aegyptiaca (L.) Willd., Tribulus terrestris L. (Ousseina, 2012) parsemée de plantes ligneuses (Maerua crassifolia Forsk., Balanites aegyptiaca (L.) Del., Acacia senegal (L.) Willd., Acacia tortilis (Forssk.) Hayne subsp. raddiana (Savi) Brenan, Salvadora persica L.., Commifora africana 
(A.Rich.) Engl.. etc (Diatta et al., 2004). Cette station couvre une superficie de 4474 ha, divisée en 5 parcs composés de 30 parcelles (Figure 1).

\section{Méthode d'inventaire des ligneux}

L'état de la végétation ligneuse de la Station a été établi à l'aide des relevés de terrain réalisés entre juillet et septembre 2009. La caractérisation du peuplement ligneux de la Station a été établie à partir de son cortège floristique, de la structure démographique des populations, du potentiel de régénération des espèces et du recouvrement.

Les mesures ont été effectuées sur quatre parcelles de superficies respectives 263 ha, 114 ha, 127 ha et 162 ha (Figure 1). Le choix de ces parcelles réside dans leur représentativité par rapport aux différentes situations physionomiques et géomorphologiques des parcs de la station ainsi que leur accessibilité en saison pluvieuse. Le taux d'échantillonnage est de $15,14 \%$.

Les relevés floristiques ont été effectués dans chaque parcelle, le long des transects parallèles distants de $500 \mathrm{~m}$ les uns des autres. Sur chaque transect, des carrés de $50 \times 50 \mathrm{~m}\left(2500 \mathrm{~m}^{2}\right)$ distants de $200 \mathrm{~m}$ sont délimités et un recensement systématique de toutes les espèces ligneuses a été réalisé à l'intérieur de ces carrés. Au total, 45 carrés ont été inventoriés. Pour chaque individu répertorié, l'espèce est déterminée au moment de l'inventaire soit par son nom latin, soit par son nom local ou à partir des ouvrages de référence. Les spécimens non identifiés sur place sont amenés au laboratoire pour identification. Pour chaque ligneux, les paramètres suivants sont mesurés:

- la hauteur totale et le diamètre à la base du tronc à $30 \mathrm{~cm}$ du sol pour établir la structure du peuplement et la surface terrière,

- les 2 diamètres perpendiculaires du houppier pour évaluer le recouvrement,

- l'identification et l'inventaire des plants juvéniles pour apprécier le potentiel de régénération des espèces (nombre de rejets des souches et des plantules). Selon Poupon (1980) les plants juvéniles sont des plants dont le diamètre du tronc à la base est inférieur à $3,5 \mathrm{~cm}$ soit $10 \mathrm{~cm}$ de circonférence. Mais pour cette étude, nous avons considéré comme plants juvéniles ceux dont le diamètre est inférieur à $4 \mathrm{~cm}$, conformément au dernier atelier d'harmonisation des méthodes tenu à Niamey du 3 au 10 août 2008.

\section{Analyse des données}

Chaque espèce est identifiée par son nom scientifique. La nomenclature quant à elle fait référence à celle de Lebrun et Stork (1991-1999) citée par Mahamane (2005). Les espèces recensées sont ensuite regroupées par type biologique, famille et genre. La classification des types biologiques fait appel à celle de Raunkiaer (1934) adaptée à l'étude de la flore tropicale par Lebrun (1947) et Trochain (1970).

L'aire de répartition phytogéographique, qui traduit les affinités chorologiques des espèces, est également mentionnée.

Les données recueillies sur les fiches de relevés ont été saisies et traitées à l'aide du logiciel Excel et les descripteurs suivants ont été déterminés:

- La diversité spécifique : analysée à l'aide des indices couramment utilisés dont la richesse floristique, la composition spécifique de la strate ligneuse, l'indice de diversité de Shannon-Weaver et l'indice d'équitabilité de Pielou.

- l'indice de valeur d'importance (IVI) déterminé pour chaque espèce (en pourcentage) est calculé par la formule de Curtis et McIntosh (Adjonou et al., 2009). Il est égal à la somme de la densité relative, de la dominance relative et de la fréquence relative. Cet indice permet d'évaluer la prépondérance spécifique de chaque espèce dans le peuplement. Il donne une mesure combinée standard de l'abondance, la dominance et la répartition de chaque espèce.

- la densité des ligneux (nombre de pieds à l'hectare ou rapport des effectifs 
rencontrés par la superficie totale des placettes en hectares) ;

- le recouvrement ligneux: $R(\%)=S h * 100 / S s$ avec Sh la surface du houppier et Ss la surface du site inventorié (placette d'inventaire);

- la surface terrière $\left(\mathrm{m}^{2} / \mathrm{ha}\right)$ par la relation: $S=\sum \pi d^{2} / 4$ où $\mathrm{d}$ est le diamètre du tronc ;

- le taux de renouvellement qui est le rapport entre l'effectif total des jeunes plants et l'effectif des plants adultes du peuplement;

- l'importance spécifique (IS) de chaque espèce dans la régénération des ligneux est le rapport entre l'effectif des jeunes plants d'une espèce et l'effectif des jeunes plants dénombrés;

- le taux de régénération : rapport entre l'effectif des jeunes plants d'une espèce et l'effectif total de l'espèce (Poupon, 1980) ;

- la structure du peuplement (répartition par classe de hauteur ou taille et de diamètre ou grosseur qui indique le niveau d'équilibre des classes d'âge et les différentes phases vécues par les populations ligneuses en termes de perturbation ou de régénération (Onana et Devineau, 2002). L'appréciation de la structure du peuplement s'est faite sur la base d'interprétation des histogrammes de distribution des ligneux dans les classes de diamètre et de hauteur en tenant compte des fréquences (\%) des individus dans le peuplement. Des courbes des tendances ont été associées aux histogrammes pour avoir une modélisation qui exprime la tendance théorique du peuplement. Neuf (9) classes de diamètre (d) et 10 classes de hauteur (h) ont été définies. Les individus sont répartis par classe de diamètre $(0$ à $4 ; 4$ à $10 ; 10$ à $15 ; 15$ à $20 ; 20$ à $25 ; 25$ à $30 ; 30$ à $35 ; 35$ à $40 ;>$ $40 \mathrm{~cm})$ et par classe de hauteur $(0$ à $1 ; 1$ à 2 ; 2 à $3 ; 3$ à $4 ; 4$ à $5 ; 5$ à $6 ; 6$ à $7 ; 7$ à $8 ; 8$ à 9 ; > $9 \mathrm{~m})$.

\section{RESULTATS}

\section{Composition floristique}

L'inventaire a permis de recenser 15 espèces réparties entre 11 genres et 10 familles (Tableau 1). Les familles les plus représentées sont les Capparaceae et Leguminosae-Mimosö̈deae (3 espèces chacune) suivie des Combretaceae (2 espèces). Les autres familles sont représentées par une seule espèce. Il s'agit des Balanitaceae, Salvadoraceae, Asclepiadaceae, Rhamnaceae, Boraginaceae, Burseraceae et Tiliaceae.

Tous les genres rencontrés sont représentés par une seule espèce, sauf les genres Acacia (3 espèces), Combretum (2 espèces) et Boscia (2 espèces).

Les types biologiques rencontrés sont essentiellement les microphanérophytes (avec 14 espèces sur les 15 dénombrées) et une seule espèce de type nanophanérophyte. Le spectre phytogéographique est dominé par les espèces à distribution africaine $(66,7 \%)$ et paléotropicale $(33,3 \%)$. Ce sont essentiellement des espèces à distribution soudano-zambézienne - saharo - sindienne $(60 \%)$ et soudano - zambéziennes $(33,3 \%)$.

\section{Densité, Contribution spécifique et diversité floristique}

Le peuplement est dominé essentiellement par Maerua crassifolia Forsk.. En effet, sur un total de 1936 individus inventoriés, 945 sont des pieds de Maerua crassifolia, soit $48,8 \%$ de contribution spécifique (Cs) et une densité (d) de 84 pieds à l'hectare. Ensuite viennent Balanites aegyptiaca avec 349 individus, Acacia raddiana avec 235 pieds, Acacia seyal Del. (105 pieds), Acacia senegal (L.) Willd. (91 pieds) et Boscia senegalensis (Pers.) Lam. ex Poir. (77 pieds).

Trois espèces que sont Maerua crassifolia (48,8\%), Balanites aegyptiaca (18\%), et Acacia raddiana $(12,1 \%)$ représentent près de $79 \%$ de l'effectif du 
peuplement (1529 individus) avec une densité respective de 84,31 et 20,9 individus à l'ha (Tableau 1). Les autres espèces contribuent individuellement pour moins de $2 \%$ au peuplement à l'exception de Boscia senegalensis, Acacia seyal et Acacia senegal qui ont une contribution spécifique de $4 \%$, $5,4 \%$ et $4,7 \%$ respectivement. L'indice de Shannon est de 2,39 bits et l'équitabilité de Pielou est égale à 0,22 . La densité globale des ligneux est de 172 pieds à l'hectare.

\section{Caractérisation du peuplement ligneux}

Le recouvrement moyen de la strate ligneuse est estimé à 12,67\%. L'évaluation de l'indice de valeur d'importance de toutes les espèces ligneuses permet de déterminer les espèces qui marquent le peuplement. Les caractéristiques de chaque espèce sont présentées dans le Tableau 2. Les espèces qui contribuent le plus au recouvrement sont : Maerua crassifolia (3,01\%), Acacia senegal (1,97\%), Acacia raddiana (1,96\%), Acacia seyal $(1,84 \%)$, Balanites aegyptiaca $(1,55 \%)$ et $B$. senegalensis $(1,42 \%)$. Les espèces prépondérantes (IVI $\geq 50$ points) sont Maerua crassifolia $(186,90)$ avec l'indice le plus élevé, Acacia raddiana (108,26), Balanites aegyptiaca $(109,41)$, Acacia senegal $(56,69)$, Acacia seyal $(73,48)$ et $B$. senegalensis $(62,18)$. La surface terrière est de $17,23 \mathrm{~m}^{2}$ à l'hectare répartie entre Maerua crassifolia $\left(6,46 \mathrm{~m}^{2} / \mathrm{ha}\right)$, Balanites aegyptiaca $(3,61$ $\left.\mathrm{m}^{2} / \mathrm{ha}\right)$, Acacia raddiana $\left(2,81 \mathrm{~m}^{2} / \mathrm{ha}\right)$, Acacia senegal (1,32 $\left.\mathrm{m}^{2} / \mathrm{ha}\right)$, Acacia seyal (1,05 $\mathrm{m}^{2} / \mathrm{ha}$ ) et Salvadora persica L. (1,05 m²/ha). Les autres espèces assurent moins de $1 \mathrm{~m}^{2} /$ ha de surface terrière. Maerua crassifolia domine largement le peuplement avec une densité relative d'environ $50 \%$ et est présente dans tous les relevés. On peut dire alors que le peuplement correspond à une formation de Maerua crassifolia avec comme espèces compagnes Balanites aegyptiaca, Acacia raddiana et Acacia senegal.

\section{Régénération et renouvellement du peuplement}

La strate juvénile ligneuse est composée de 14 espèces réparties dans 11 genres et 10 familles. Au cours de cet inventaire, seule Combretum glutinosum Pers. ex DC. est absente dans la population juvénile. Cette espèce est représentée par 2 pieds adultes dans le peuplement. Les plants juvéniles (toutes espèces confondues) constituent $39,7 \%$ du peuplement (768 pieds). Toutefois, les taux de régénération et de renouvellement sont très variables selon l'espèce (Tableau 3). En prenant en compte l'importance spécifique des espèces, ce sont Maerua crassifolia, Balanites aegyptiaca et Acacia raddiana qui dominent le peuplement juvénile $\quad(47,9 \%, \quad 17,4 \%$ et $14,1 \%$ respectivement).

La capacité ou taux de renouvellement des populations de Boscia angustifolia A. Rich., B. senegalensis, Commiphora africana (A. Rich.) Engl.,Grewia tenax (Forsk.) Fiori et Calotropis procera (Ait) R. Br. sont respectivement de $165,5 \%, 210 \%, 112,5 \%$, $116,7 \%$ et $140 \%$. La population de ces 5 espèces présente plus de jeunes plants que d'adultes et semble régénérer mieux que les autres espèces.

Les populations de Combretum aculeatum Vent. et Cordia sinensis Lam. sont constituées uniquement de plants juvéniles ( 2 pieds chacune).

\section{Structure du peuplement Distribution par classe de hauteur}

La distribution des ligneux par classe de hauteur permet d'appréhender la structure verticale des ligneux de la station. Le peuplement ligneux présente une distribution par classes de hauteur en «dents de scie » qui s'ajuste à une courbe de tendance polynomiale de degré $3\left(\mathrm{R}^{2}=0,94\right)$ et traduit une répartition erratique de la hauteur des ligneux dans les différentes classes (Figure 2-A). $y=0,11 x^{3}-1,62 x^{2}+2,90 x+22,74$ 
avec $\mathrm{R}^{2}=0,94$.

L'essentiel des ligneux se trouve dans les classes de hauteur de [0-1[ mètre $(24,7 \%)$, suivie par la classe de hauteur [1-2[ mètres $(21,7 \%)$. Ces deux classes précèdent les classes [2-3[ mètres et [3-4[ mètres de hauteur. Les autres classes de hauteur comprises entre 4 et $6 \mathrm{~m}$ représentent $12,3 \%$ $\mathrm{du}$ peuplement. Les classes les moins représentées sont celles supérieures à $6 \mathrm{~m}$ avec $1,3 \%$ des individus.

La structure de la population des ligneux dominants est illustrée par les Figures 2-B, C et D. La Figure 2-B indique que les individus de Maerua crassifolia sont concentrés dans les strates de 0 à $4 \mathrm{~m}$, avec plus de $91 \%$ des individus. Leur distribution s'ajuste à une courbe de tendance polynomiale de degré 3 avec un coefficient de détermination $\left(\mathrm{R}^{2}\right)$ proche de 1 .

La distribution de la population de Balanites aegyptiaca (Figure 2-C) s'adapte à une courbe de tendance polynomiale de degré 3 en forme de "S" renversé traduisant une concentration des individus dans une strate:

$\mathrm{y}=0,16 \mathrm{x}^{3}-2,63 \mathrm{x}^{2}+8,72 \mathrm{x}+13,72$

avec $\mathrm{R}^{2}=0,97$.

En effet, les individus de cette espèce sont essentiellement concentrés dans la strate 1 à 4 mètres de haut et totalisent $62,6 \%$ de la population.

La structure en classes de hauteur de la population de Acacia raddiana présente une distribution en "dents de scie", qui s'ajuste à une fonction polynomiale de degré 4 traduisant une répartition très irrégulière des individus dans les différentes strates (Figure $1-\mathrm{D})$.

$y=0,047 x^{4}-0,85 x^{3}+5,36 x^{2}-16,69 x+38,69$ avec $\mathrm{R}^{2}=0,84$.

Les individus des classes de 0 à $2 \mathrm{~m}$ et de 3 à 4 m sont majoritaires (70\%), suivis par ceux des classes de 2 à $3 \mathrm{~m}(12 \%)$ et 5 à $6 \mathrm{~m}$ $(10 \%)$. Les classes 4 à 5 et $\geq$ à $6 \mathrm{~m}$ représentent respectivement $6,5 \%$ et $1,5 \%$ du peuplement.

Les individus ayant une hauteur supérieure à $6 \quad \mathrm{~m}$ sont constitués essentiellement de Balanites aegyptiaca (9 pieds), Acacia seyal (5 pieds), Maerua crassifolia (3 pieds), Acacia raddiana (3 pieds), Acacia senegal (2 pieds) et Combretum glutinosum (1 pied).

\section{Distribution par classes de diamètre}

La distribution du peuplement ligneux de la Station par classes de diamètre (Figure 3-A) s'ajuste à une fonction exponentielle en forme de " $\mathrm{L}$ " avec un coefficient de détermination $\mathrm{R}^{2}=0,94$ et dont l'équation est : $\mathrm{y}=70,22 \mathrm{e}^{-0,59 \mathrm{x}}$. Cette structure est caractérisée par une répartition décroissante des individus des petites classes de diamètre vers les grandes classes. La même tendance est observée pour les populations des espèces dominantes de la Station Maerua crassifolia, Balanites aegyptiaca et Acacia raddiana (Figures 3- B, C et D).

La grosseur des arbres et arbustes estimée à partir du diamètre du tronc à la base varie entre 0 et $63,1 \mathrm{~cm}$. La Figure 3 montre que les jeunes plants (diamètre inférieur à 4 $\mathrm{cm}$ ) représentent $38,4 \%$ du peuplement de la Station. Les arbres de gros diamètres (plus de $30 \mathrm{~cm}$ de diamètre) n'en constituent que 2,1\% et ceux des classes moyennes (4 à $30 \mathrm{~cm}$ de diamètre) représentent $59,6 \%$ des ligneux recensés.

\section{Relation allométrique de la croissance des ligneux}

La relation allométrique de croissance qui existe entre la hauteur et le diamètre (Figure 4-A'") s'ajuste à une fonction logarithmique dont l'équation est :

$\mathrm{y}=1,10 \ln (\mathrm{x})+0,58$ avec $\mathrm{R}^{2}=0,61$

Ce graphique montre que la plupart des ligneux dont la hauteur est comprise entre 2 et 
$6 \mathrm{~m}$ ont un diamètre inférieur à $30 \mathrm{~cm}$. La dispersin importante du nuage de points montre une diversité de dimensions et de formes des plantes, même au sein de la même espèce (Figure 4- B', C', et D'). Toutefois, la hauteur ne crôit pas indéfiniment en fonction du diamètre. En effet, chez les individus de gros diamètre, il semble exister une relation entre la croissance en hauteur et le développement diamétral du tronc des ligneux. Celui-ci peut continuer à croître tandis que la croissance en hauteur se stabilise autour de $9 \mathrm{~m}$.

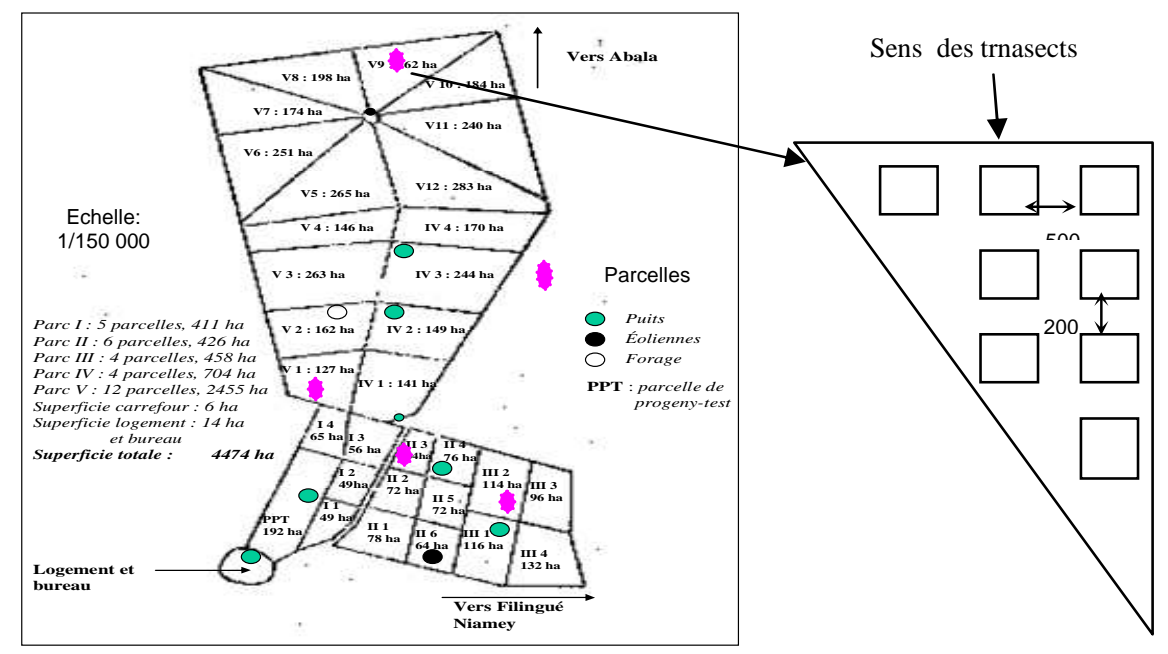

Figure 1: Schéma du parcellement de la Station de Toukounous, parcelles inventoriées.

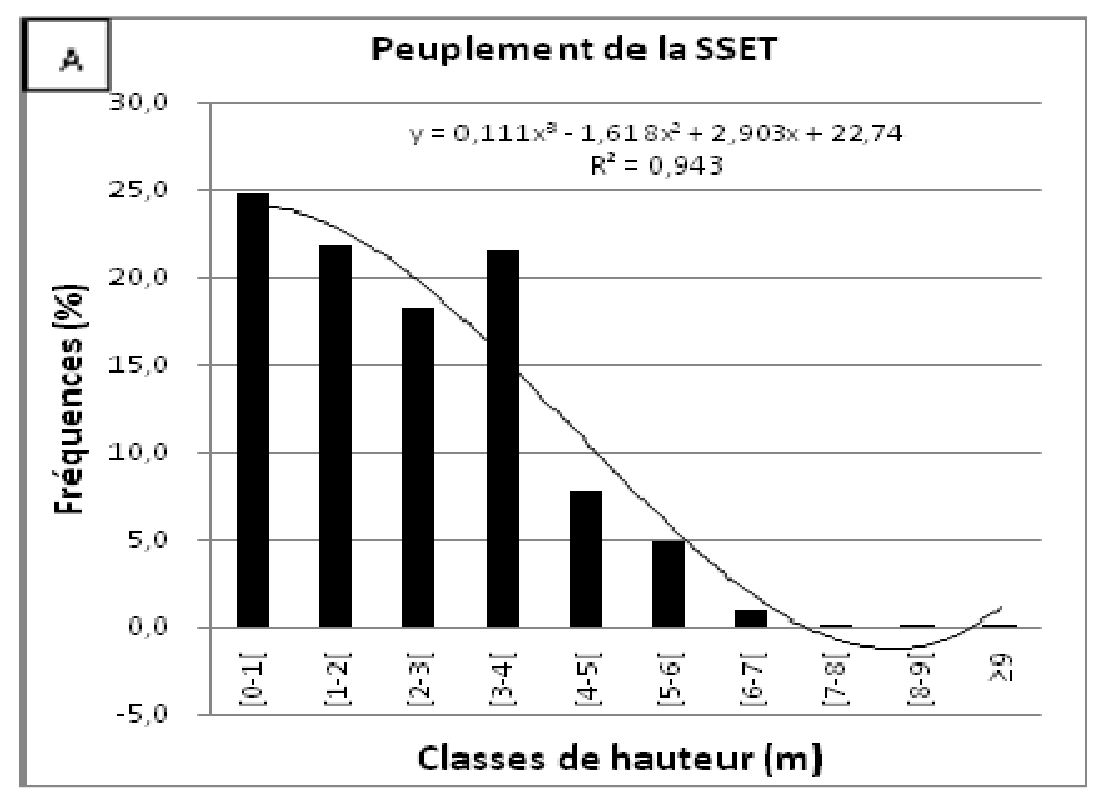



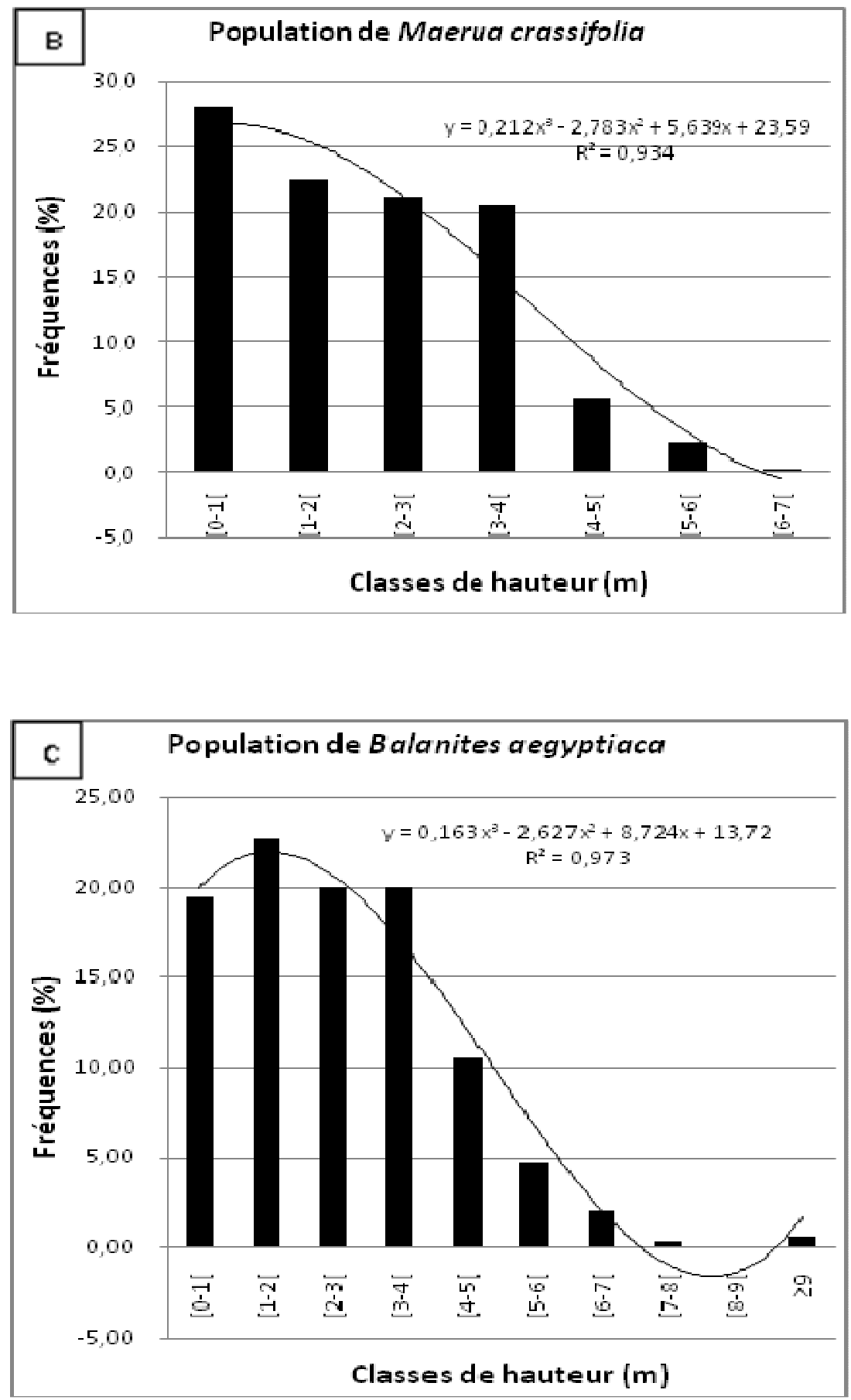


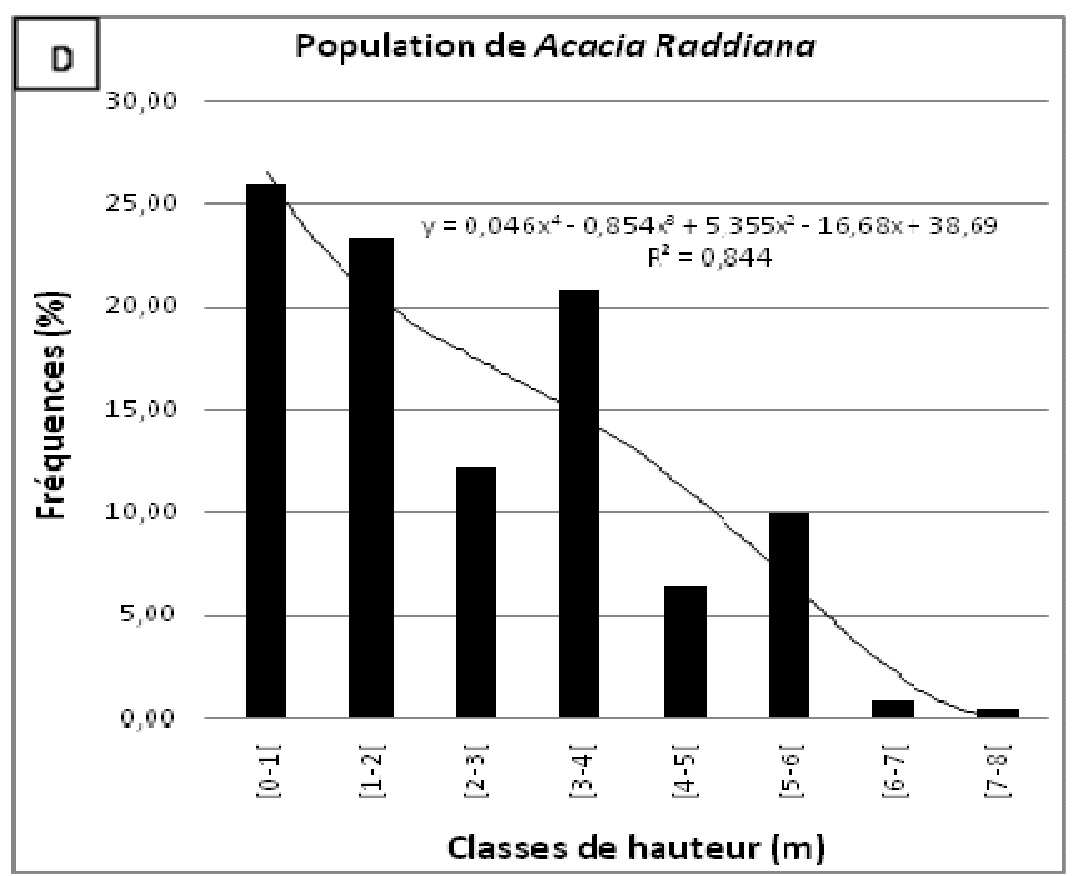

Figure 2 : Distribution des fréquences $(\%)$ de la végétation ligneuse par classes de hauteur. A : Hauteur du peuplement ; B : Hauteur Population de Maerua crassifolia ; C : Hauteur Population de Balanites aegyptiaca ; D : Hauteur Population de Acacia raddiana.

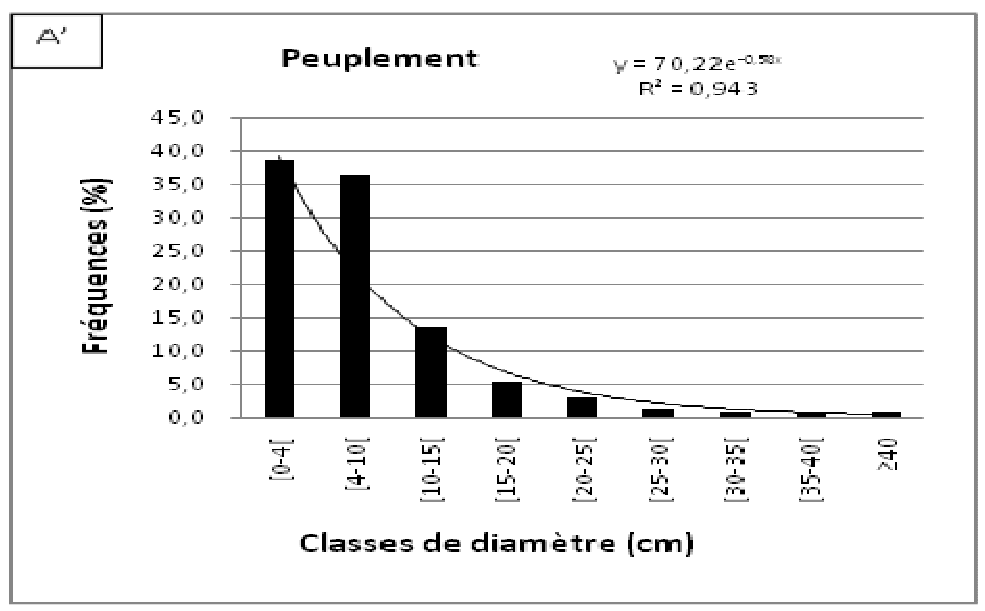


S. OUSSEINA et al. / Int. J. Biol. Chem. Sci. 9(2): 910-926, 2015
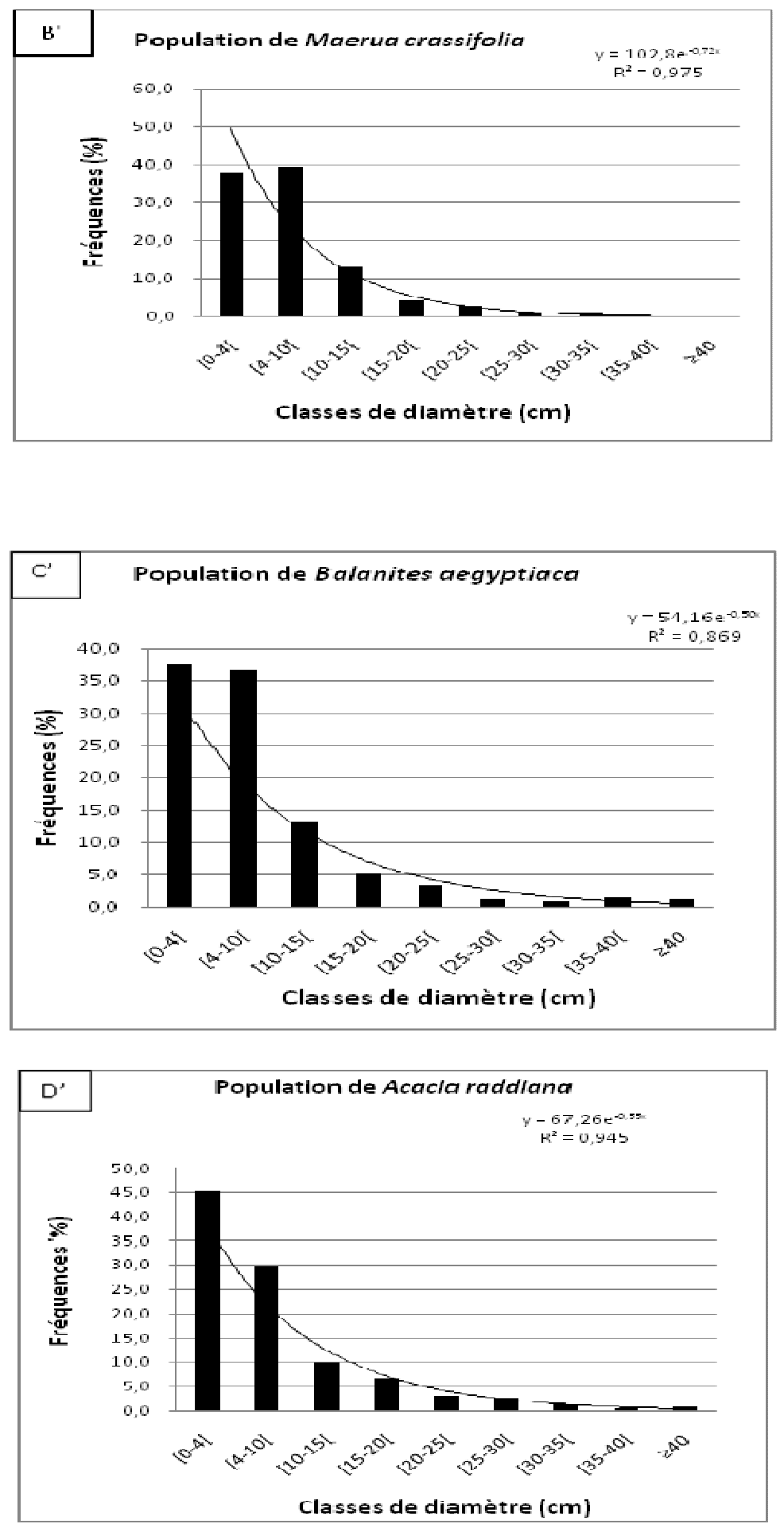

Figure 3 : Distribution des fréquences $(\%)$ de la végétation ligneuse par classe de diamètre $(\mathrm{cm})$. A': Diamètre du peuplement ; B' : Diamètre Population de Maerua crassifolia ; C': Diamètre Population de Balanites aegyptiaca ; D' : Diamètre Population de Acacia raddiana. 
S. OUSSEINA et al. / Int. J. Biol. Chem. Sci. 9(2): 910-926, 2015
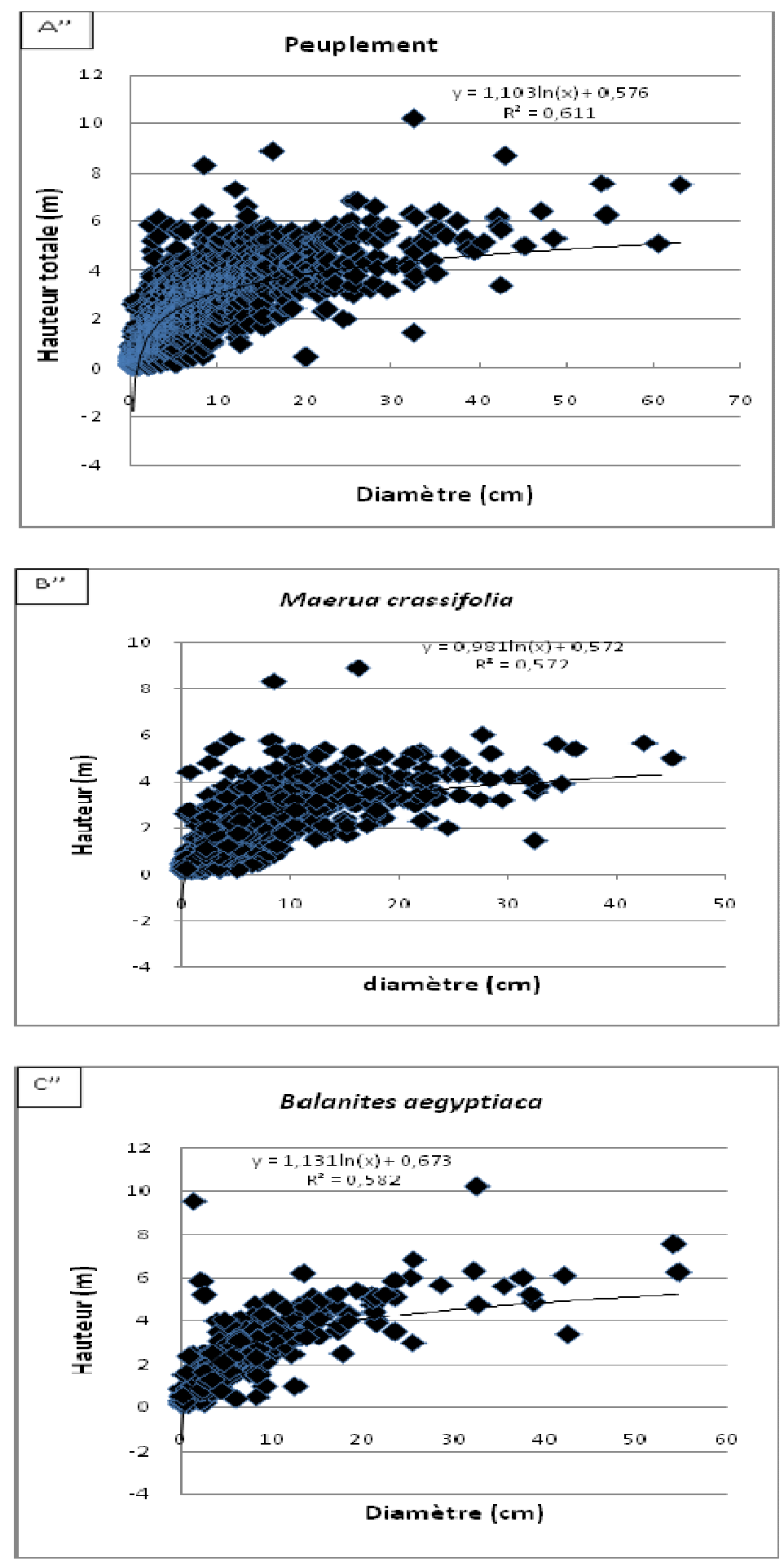


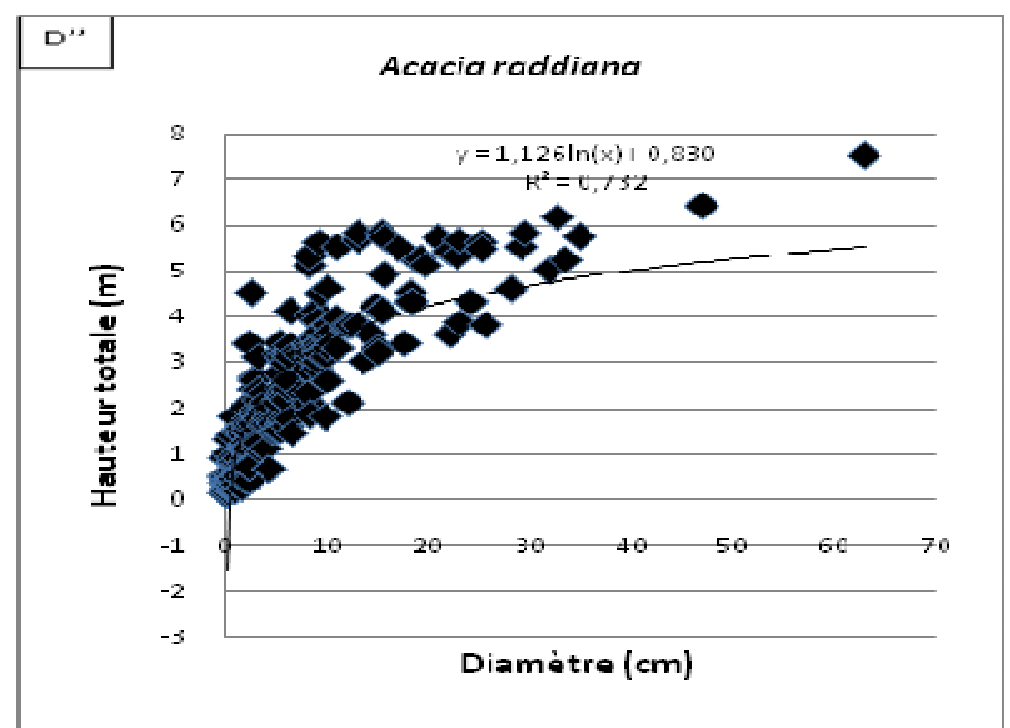

Figure 4 : Courbes de régression traduisant la relation de la croissance de la hauteur en fonction du diamètre. A": Peuplement ; B" : Population de Maerua crassifolia ; C": Population de Balanites aegyptiaca ; D": Population de Acacia raddiana.

Tableau 1 : Types biologiques, familles, genres, effectif (fréquence absolue FA), contribution spécifique $(\mathrm{Cs} \%)$ et densité ( $\mathrm{d}$ pieds/ha) des espèces ligneuses recensées.

\begin{tabular}{|c|c|c|c|c|c|c|}
\hline Type biologique & Familles & Genres & Espèces & FA & Cs & d \\
\hline \multirow{14}{*}{ 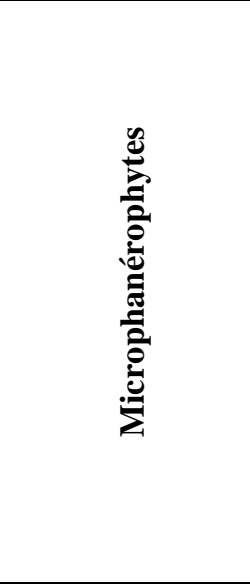 } & \multirow{3}{*}{ Capparaceae } & Maerua & Maerua crassifolia & 945 & 48,8 & 84,0 \\
\hline & & \multirow{2}{*}{ Boscia } & Boscia angustifolia & 13 & 0,7 & 1,2 \\
\hline & & & Boscia senegalensis & 77 & 4,0 & 6,8 \\
\hline & Balanitaceae & Balanites & Balanites aegyptiaca & 349 & 18,0 & 31,0 \\
\hline & Boraginaceae & Cordia & Cordia sinensis & 2 & 0,1 & 0,2 \\
\hline & Burceraceae & Commiphora & Commiphora africana & 34 & 1,8 & 3,0 \\
\hline & \multirow{3}{*}{ Mimosaceae } & \multirow{3}{*}{ Acacia } & Acacia raddiana & 235 & 12,1 & 20,9 \\
\hline & & & Acacia senegal & 91 & 4,7 & 8,1 \\
\hline & & & Acacia seyal & 105 & 5,4 & 9,3 \\
\hline & Salvadoraceae & Salvadora & Salvadora persica & 31 & 1,6 & 2,8 \\
\hline & Ascleropédiaceae & Calotropis & Calotropis procera & 12 & 0,6 & 1,1 \\
\hline & Rhamnaceae & Ziziphus & Ziziphus mauritiana & 7 & 0,4 & 0,6 \\
\hline & \multirow{2}{*}{ Combrétaceae } & \multirow{2}{*}{ Combretum } & Combretum aculaetum & 2 & 0,1 & 0,2 \\
\hline & & & Combretum glutinosum & 2 & 0,1 & 0,2 \\
\hline $\begin{array}{l}\text { Nano- } \\
\text { phanérophytes }\end{array}$ & Tiliaceae & Grewia & Grewia tenax & 31 & 1,6 & 2,8 \\
\hline Total & 10 & 11 & 15 & 1936 & 100,0 & 172,1 \\
\hline
\end{tabular}


Tableau 2 : Caractéristiques de la végétation ligneuse (degré de couverture (DC) et indice de valeur d'importance (IVI) des espèces ligneuses recensées).

\begin{tabular}{lcccccc}
\hline Espèces & $\begin{array}{c}\text { DC } \\
(\%)\end{array}$ & ST $\left(\mathbf{m}^{\mathbf{2} / \mathbf{h a})}\right.$ & $\begin{array}{c}\text { Dominance } \\
\text { relative }\end{array}$ & $\begin{array}{c}\text { Densité } \\
\text { relative } \mathbf{\%})\end{array}$ & $\begin{array}{c}\text { Fréquence } \\
\text { relative } \mathbf{\%})\end{array}$ & IVI \\
\hline Maerua crassifolia & 3,01 & 6,46 & 37,50 & 49,40 & 100,00 & 186,90 \\
Acacia raddiana & 1,96 & 2,81 & 20,96 & 18,41 & 68,89 & 108,26 \\
Balanites aegyptiaca & 1,55 & 3,61 & 16,31 & 10,87 & 82,22 & 109,41 \\
Acacia senegal & 1,97 & 1,32 & 0,87 & 2,48 & 53,33 & 56,69 \\
Acacia seyal & 1,84 & 1,05 & 7,66 & 5,82 & 60,00 & 73,48 \\
Boscia senegalensis & 1,42 & 0,15 & 6,09 & 7,19 & 48,89 & 62,18 \\
Salvadora persica & 0,54 & 1,05 & 0,17 & 0,86 & 24,44 & 25,47 \\
Grewia tenax & 0,03 & 0,03 & 1,18 & 1,37 & 20,00 & 22,55 \\
Commiphora africana & 0,17 & 0,20 & 6,09 & 1,97 & 28,89 & 36,95 \\
Boscia angustifolia & 0,01 & 0,04 & 0,23 & 0,51 & 13,33 & 14,08 \\
Ziziphus mauritiana & 0,02 & 0,03 & 0,01 & 0,43 & 4,44 & 4,88 \\
Calotropis procera & 0,01 & 0,00 & 0,01 & 0,00 & 2,22 & 2,23 \\
Cordia sinensis & 0,00 & 0,04 & 0,23 & 0,00 & 4,44 & 4,68 \\
Combretum glutinosum & 0,14 & 0,43 & 0,17 & 0,51 & 8,89 & 9,58 \\
Combretum aculeatum & 0 & 0,00 & 2,50 & 0,17 & 2,22 & 4,89 \\
Total & $\mathbf{1 2 , 6 7}$ & $\mathbf{1 7 , 2 3}$ & $\mathbf{1 0 0 , 0 0}$ & $\mathbf{1 0 0 , 0 0}$ & - & - \\
\hline
\end{tabular}

Tableau 3 : Caractéristiques écologiques de la population de plants juvéniles recensés en fonction des espèces ligneuses.

\begin{tabular}{lcccccc}
\hline \multicolumn{1}{c}{ Espèces } & $\begin{array}{c}\text { Fréquence } \\
\text { absolue } \\
\text { peuplement } \\
\text { (pieds) }\end{array}$ & $\begin{array}{c}\text { Fréquence } \\
\text { absolue } \\
\text { jeunes } \\
\text { plants } \\
\text { (pieds) }\end{array}$ & $\begin{array}{c}\text { Importance } \\
\text { spécifique } \\
\text { IS }(\boldsymbol{\%})\end{array}$ & $\begin{array}{c}\text { Densité } \\
\text { absolue } \\
\text { (pieds/ha) }\end{array}$ & $\begin{array}{c}\text { Taux de } \\
\text { Régénération } \\
(\%)\end{array}$ & $\begin{array}{c}\text { Taux de } \\
\text { Renouvellement } \\
(\%)\end{array}$ \\
\hline Maerua crassifolia & 945 & 368 & 47,9 & 32,7 & 38,9 & 63,8 \\
Balanites aegyptiaca & 349 & 134 & 17,4 & 11,9 & 38,4 & 62,3 \\
Acacia raddiana & 235 & 108 & 14,1 & 9,6 & 46,0 & 85,0 \\
Boscia senegalensis & 77 & 48 & 6,3 & 4,3 & 62,3 & 165,5 \\
Acacia senegal & 91 & 23 & 3,0 & 2,0 & 25,3 & 33,8 \\
Acacia seyal & 105 & 21 & 2,7 & 1,9 & 20,0 & 25,0 \\
Grewia tenax & 31 & 21 & 2,7 & 1,9 & 67,7 & 210,0 \\
Commiphora africana & 34 & 18 & 2,3 & 1,6 & 52,9 & 112,5 \\
Salvadora persica & 31 & 8 & 1,0 & 0,7 & 25,8 & 34,8 \\
Boscia angustifolia & 13 & 7 & 0,9 & 0,6 & 53,8 & 116,7 \\
Calotropis procera & 12 & 7 & 0,9 & 0,6 & 58,3 & 140,0 \\
Combretum aculeatum & 2 & 2 & 0,3 & 0,2 & $*$ & $*$ \\
Cordia sinensis & 2 & 2 & 0,3 & 0,2 & $*$ & $*$ \\
Ziziphus mauritiana & 7 & 1 & 0,1 & 0,1 & 14,3 & 16,7 \\
Combretum glutinosum & 2 & 0 & 0,0 & 0,0 & 0,0 & 0,0 \\
Total & 1936 & 768 & 100,0 & 68,3 & 39,7 & 65,8 \\
\hline
\end{tabular}




\section{DISCUSSION}

Analyse de la flore

La végétation ligneuse renferme 15 espèces et est dominée essentiellement par la famille des Capparaceae (Maerua crassifolia, Boscia senegalensis, Bossia angustifolia) et celle des Leguminosae-Mimosoïdeae (Acacia raddiana, Acacia senegal et Acacia seyal). Ces deux familles constituent avec la famille des Balanitaceae, la composante la plus importante des ligneux de la Station. Les espèces appartenant à ces familles sont du domaine saharo-sahélien comme l'a souligné Arbonnier (2000). La strate ligneuse est de densité faible (172 pieds à l'hectare) et la hauteur est inférieure à 10 mètres. Cette faible densité a également été signalée dans le même peuplement ligneux de la Station de Toukounous par Diatta (2008) et Douma et al. (2007) avec respectivement une densité de 188 et 178 pieds à l'hectare. Les microphanérophytes qui dominent ce peuplement ligneux ont une stratégie de compétition optimale due à leur longévité, leur taille et leur tolérance écologique (Grime, 1977). Ce sont des espèces parfaitement adaptées au milieu Saharo-sahélien comme en témoigne leur affinité chorologique. Certaines espèces présentent une densité relativement plus élevée (Maerua crassifolia (84 pieds/ha), Balanites aegyptiaca (31 pieds/ha), Acacia raddiana (20,9 pieds/ha) et dominent le peuplement en raison de leur parfaite adaptation au milieu. Lorsqu' on compare ces densités avec les résultats de Diatta (2008) ou ceux de Douma et al. (2007), on constate que la densité de Maerua crassifolia a diminué, celle de Balanites aegyptiaca n'a pas varié alors que celle d'Acacia raddiana a augmenté. Ainsi, on assiste à une baisse de l'effectif de Maerua crassifolia, espèce dominante du peuplement ligneux et la plus appréciée par les animaux, face à l'augmentation de l'effectif d'Acacia raddiana.

La valeur de l'indice de diversité indique que le peuplement est diversifié. Cette hétérogénéité peut s'expliquer par les facteurs type de sol, durée de la concentration des eaux de pluies et topographique (Douma et al., 2007), qui jouent un rôle essentiel dans la redistribution de l'eau dans le milieu. En effet, selon Arbonnier (2000), la végétation arbustive du secteur saharien est circonscrite au fond des dépressions qui correspondent à des axes de drainage. Comme l'ont aussi fait remarqué plusieurs auteurs dans d'autres régions (Diouf et al., 2002), le paysage de la station est une zone couverte par un tapis herbacé présentant des plages dénudées par endroits et parsemé d'espèces ligneuses $(\leq 10$ $\mathrm{m}$ de haut) concentrées le plus souvent au niveau des dépressions. La valeur de l'indice d'importance de toutes les espèces ligneuses et celle de l'indice d'équitabilité de Pielou faible $(0,22)$ confirment la dominance d'une espèce dans le peuplement. Douma et al. (2007) ont d'ailleurs défini ce peuplement comme étant une formation à Maerua crassifolia. Cette dénomination qui prend en compte plusieurs paramètres descriptifs ne contredit pas celle de Achard et Chanono (1995) qui assimilent le peuplement à une formation à Maerua crassifolia et Balanites aegyptiaca en tenant compte d'un seul paramètre (distribution des individus par classe de hauteur).

La structure verticale du peuplement présente une distribution en «dents de scie ». Ce type de distribution traduit une répartition irrégulière des individus dans les différentes classes de hauteur. Cette situation s'observe dans un peuplement dégradé ou instable caractérisé par une absence ou une très faible proportion d'individus dans une ou plusieurs classes (Ouédraogo, 2006). Elle peut être une des conséquences des sécheresses récurrentes qui sévissent au Sahel conjuguées aux effets des fortes pressions sur les ressources fourragères (Cornet et al., 2002 ; Lhoste, 2007 ; Ganaba et al., 2005; Ali et al., 2008; Ali et Lebel, 2009).

La structure diamétrale est caractérisée par la prédominance des individus de petits diamètres. Cette structure classique est souvent observée pour les écosystèmes forestiers non perturbés (Adjonou et al., 
2009). La structure des classes de grosseur des arbres qui s'ajuste à une loi exponentielle décroissante témoigne d'une population en équilibre sur le plan écologique comme l'a déjà rapporté Diatta (2008) sur la population de Maerua crassifolia dans le même peuplement. D'ailleurs, cette espèce domine le peuplement sur lequel elle imprime sa dynamique. Les individus de gros diamètre sont rares dans ce peuplement $(2,1 \%)$. Cette rareté traduit l'incapacité des arbres à croître normalement en épaisseur suite aux déficits hydriques cumulés. La relation allométrique qui existe entre la hauteur et le diamètre des ligneux recensés s'exprime faiblement et s'ajuste à une fonction logarithmique dont le coefficient de détermination est égal à 0,61 . Toutefois, la hauteur ne croît pas indéfiniment en fonction du diamètre. En effet, chez les individus de gros diamètre, il semble exister une relation entre la croissance en hauteur et le développement du tronc des ligneux. Celuici peut continuer à croître tandis que la croissance en hauteur se stabilise autour de 9 $\mathrm{m}$. La concentration de la plupart des ligneux dénombrés dans les classes de hauteur comprises entre 2 et 6 mètres conforme aussi l'appartenance de la station au domaine sahélien (Diouf et al., 2002).

\section{Régénération et renouvellement du peuplement}

La régénération est un mode de reproduction d'un peuplement forestier. Elle peut être naturelle à partir des semences ou de rejets, ou artificielle par semis ou plantation. L'étude de la régénération constitue un moyen efficace d'appréciation de la reconstitution de la végétation naturelle (Yaméogo et al., 2006). La densité de régénération est globalement faible (39,7 pieds/ha). Aussi, la densité de la population juvénile reste très faible $(68,3$ pieds/ha). Le faible taux de régénération pourrait être expliqué par la vulnérabilité des jeunes plants aux facteurs de dégradation, telles que la sécheresse et la pâture. En effet, les jeunes sujets connaissent des problèmes de croissance comme le montre l'importance des individus de faible diamètre au détriment des ligneux à gros diamètre. Le recrutement des individus dans les classes supérieures est soumis à la pression de pâturage et aux aléas climatiques (faible réserve en eau du sol, fortes chaleurs,...). Sokpon et al. (2006) dans le Nord-Bénin et Sawadogo et al. (2002) dans les savanes soudaniennes du Burkina Faso ont évoqué l'effet de la pâture sur les jeunes plants. L'irrégularité et la précarité des pluies enregistrées au Sahel pendant ces dernières décennies a pour conséquences la diminution du stock semencier et la baisse du niveau des nappes alluviales (Hervouet, 1974 rapporté par Douma et al., 2007) pouvant entraîner une baisse de production de plants par germination.

La capacité de reproduction des espèces dans les conditions naturelles (renouvellement des espèces) est un concept lié à la fois à l'importance de la populationmère et à l'effectif des jeunes plants. A la Station de Toukounous, le taux de renouvellement des espèces est très varié. Dans les populations de Maerua crassifolia, Balanites aegyptiaca, Acacia raddiana, Acacia senegal, Acacia seyal, et Salvadora persica, la proportion de jeunes individus est faible par rapport aux individus âgés. Cela traduit un vieillissement de la population de ces espèces. Toutefois, ce groupe est composé d'espèces les plus appétées par les bovins. Pendant les périodes de déficit fourrager herbacé (saison sèche), les ligneux sont très sollicités par le bétail. Les jeunes plants sont prioritairement broutés pendant la période sèche et leur survie se trouve menacée. Ce phénomène prépondérant dans la zone étudiée augmente le taux de mortalité des jeunes sujets (Achard et Chanono, 1995). Les espèces les moins accessibles (Grewia tenax, Commiphora africana), les moins appétées (Boscia angustifolia, Boscia senegalensis) ou pas appétées (Calotropis procera à l'état vert) ont un taux de renouvellement plus élevé, c'est-à-dire que les plants juvéniles sont plus nombreux que les plants adultes. La population de ces espèces semble être en 
phase de rajeunissement. Ce processus peut se poursuivre si les conditions climatiques s'y prêtent. En effet, comme l'ont déjà rapporté certains auteurs, les perturbations écologiques de ces dernières décennies ont eu pour conséquences la régression de certaines espèces comme Sclerocarya birrea (A. Rich.) Hochst., Combretum glutinosum et Acacia senegal dans les stations à bilan hydrique médiocre (Cornet, 1981), la concentration d'autres espèces (Acacia senegal, Ziziphus mauritiana Lam.) dans les points bas (Piot et al., 1980) et des espèces en pleine expansion (Boscia senegalensis, Balanites aegyptiaca, Calotropis procera) (Akpo et Grouzis, 1996).

Combretum aculeatum et Cordia sinensis ne sont représentées que par des individus jeunes (2 chacune) dans le peuplement. On peut supposer que ce sont des espèces nouvelles dans les parcelles inventoriées où du moins elles sont menacées. Ces deux espèces sont d'ailleurs absentes des inventaires réalisés par Diatta (2008) et Douma et al. (2007).

\section{Conclusion}

Cette étude a permis de faire une situation de l'état et des caractéristiques du peuplement ligneux de la station de Toukounous. La végétation ligneuse est riche de 15 espèces réparties dans 11 genres et 10 familles. Ce sont des espèces à distribution africaine et paléotropicales essentiellement constituées de micorphanérophytes. Les familles les plus importantes sont les Capparaceae et les Mimosaceae. Les espèces les plus abondantes dans le peuplement sont Maerua crassifolia, Balanites aegyptiaca et Acacia raddiana. L'effectif de Maerua crassifolia, espèce dominante et très appétée par le bétail tend à baisser comparativement à Balanites aegyptiaca et Acacia raddiana. La structure du peuplement traduit une situation d'un milieu perturbé. La régénération et le renouvellement du peuplement sont faibles. Les espèces les plus appétées sont le plus souvent broutées à l'état de plants juvéniles compromettant ainsi leur évolution vers les plants adultes. Des mesures doivent être prises pour protéger les jeunes plants et favoriser leur émergence afin d'assurer au bétail une source de nutriments pendant la période de soudure. Il est aussi nécessaire de poursuivre les investigations pour étudier le fonctionnement $\mathrm{du}$ peuplement ligneux en relation avec les variations des paramètres climatiques et des états de surfaces.

\section{REFERENCES}

Achard F, Chanono M. 1995. Un système d'élevage performant bien adapté à l'aridité à Toukounous, dans le Sahel nigérien. Sécheresse, 2(6): 215-222.

Adjonou K, Bellefontaine R, Kokou K. 2009. Les forêts claires du Parc national OtiKéran au Nord-Togo : structure, dynamique et impacts des modifications climatiques récentes. Sécheresse, 20(1): e1-e10.

Akpo LE, Grouzis M. 1996. Influence du couvert sur la régénération de quelques espèces ligneuses sahéliennes (NordSénégal, Afrique occidentale). Webbia 50(2): 247-263.

Ali A, Lebel T. 2009. Recent trends in the Central and Western Sahel rainfall regime (1990-2007). Journal of Hydrology, 375: 52-64.

Ali A, Lebel T, Amani A. 2008. Signification et usage de l'indice pluviométrique au Sahel. Sécheresse 19(4): 227-235.

Arbonnier M. 2000. Arbres, arbustes et lianes des zones sèches d'Afrique de l'Ouest. CIRAD MNHN-UICN, $542 \mathrm{p}$.

Ciofolo I. 1995. West Africa's last giraffes: the conflict between development and conservation. Journal of Tropical Ecology, 11: 577-588.

Cornet A. 1981. Le bilan hydrique et son rôle dans la production de la strate herbacée de quelques phytocénoses sahéliennes au Sénégal. Doct. Ing., USTL, Montpellier, $353 \mathrm{p}$.

Cornet A, Lhoste P, Toutain B. 2002. Évaluation et durée des actions de lutte contre la désertification. Impacts 
environnementaux, sociaux et économiques. In Lutte contre la désertification dans les projets de développement : un regard scientifique sur l'expérience de l'AFD en Afrique sub-saharienne et au Maghreb. Cornet A, Jouve P, Corbier-Barthaux C (coord.). CSFD/AFD : Paris ; 139-150.

Diatta S. 2008. Modes de propagation d'un ligneux fourrager sahélien, Maerua crassifolia forsk. Thèse de doctorat en biologie végétale $\left(3^{\mathrm{ème}}\right.$ cycle) Option : Ecologie. Université Check Anta Diop. Dakar, 103 pp.

Diatta S, Douma S, Chanono M, Banoin M, Kaboré-Zoungrana CY, Toudou A, Akpo LE. 2004. Caractéristiques de Maerua crassifolia Forsk., ligneux fourrager des terres de parcours sahéliennes (Toukounous - Filingué, Niger). Rev.afr. Santé et Productions Animales, 2(2): 148-153.

Diouf M, Akpo LE, Rocheteau A, Do F, Goudiaby V, Diagne A L. 2002. Dynamique du peuplement ligneux d'une végétation sahélienne au NordSénégal (Afrique de l'ouest). Journal des Sciences, 2(1): 1-10.

Douma S, Diatta S, Kabore-Zoungrana CY, Banoin M, Akpo LE. 2007. Caractérisation des terres de parcours sahéliennes : typologie du peuplement ligneux de la Station sahélienne Expérimentale de Toukounous au Niger. Journal des Sciences, 7: 1-16.

FAO. 2007. Situation des Forêts du Monde. Organisation des Nations Unies pour l'Alimentation et l'Agriculture : Rome ; 2007.

Fournier A. 1991. Phénologie, Croissance et Production Végétales dans Quelques Savanes d'Afrique de l'Ouest. ORSTOM : Paris, 312 p.

Ganaba S, Ouadba JM, Bognounou O. 2005. Exploitation traditionnelle des végétaux spontanés en région sahélienne du Burkina Faso. VertigO - La Revue en Sciences de l'Environnement, $6(2)$.
Ganaba S, Guinko S. 1995. Morphologie et rôle des structures racinaires dans la mortalité de Pterocarpus lucens Lepr. dans la région sahélienne de la mare d'Oursi (Burkina Faso). Etudes sur la Flore et la végétation du Burkina Faso et des pays avoisinants 2: 15-24.

Grime JP. 1977. Evidence for the existence of three primary strategies in plants and its relevance to ecological and evolutionary theory. Amer. Nat., 111: 1169-1194.

Grouzis M. 1995. Les végétations annuelles. In Pastoralisme: Troupeaux, Espaces et Sociétés. Daget P, Godron M (eds). Ouvrage collectif AUPELF/UREF: Hatier.

Le Barbé L, Lebel T. 1997. Rainfall climatology of the HAPEX-Sahel region during the years 1950-1990. Journal of Hydrology, 188-189; 43-73.

Lebrun J. 1947. La végétation de la plaine alluviale au sud du Lac Edouard. Inst. Parcs Nat. Congo Belge, Exp. Parcs Nat. Albert. Mission Lebrun (19371938) 1, 800p.

Lhoste Ph. 2007. Sociétés pastorales et désertification au Sahel. Rev. Bois et Forêts des Tropiques, 293(3): 49-59.

Mahamane A. 2005. Etudes floristique, phytosociologique phytogéographique de la végétation du parc régional du $\mathrm{W}$ Niger. Thèse présentée á l'Université Libre de Bruxelles pour obtenir le titre de Docteur Ingénieur, $516 \mathrm{p}$.

Onana J, Devineau JL. 2002. Afzelia africana Smith ex Persoon dans le Nord Cameroun. Etat actuel des peuplements et utilisation pastorale. Elev. Med. Vet. Pays Trop., 55(1): 39-45.

Ouédraogo A. 2006. Diversité et dynamique de la végétation ligneuse de la partie orientale du Burkina faso. Thèse de Doctorat, Université de Ouagadougou (Burkina Faso), p.196.

Ousseina S. 2012. Etude des ressources fourragères de la Station Sahélienne 
Expérimentale de Toukounous: diversité, structure, dynamique, qualité et disponibilité. Thèse de Doctorat, Université Abdou Moumouni Niamey (Niger), 119 p.

Ozer P, Erpicum M. 1995. Méthodologie pour une meilleure représentation spatiotemporelle des fluctuations pluviométriques observées au Niger depuis 1905: Secheresse, 6(1): 103108.

Piot J, Nebout JP, Nano T, Toutain B. 1980. Utilisation des ligneux sahéliens par les herbivores domestiques CTFT/ IEMVT, Ouagadougou, Rapp. Multigr.

Poupon H. 1980. Structure dynamique de la strate ligneuse d'une steppe sahélienne au Nord du Sénégal. Travaux et documents de l'ORSTOM n ${ }^{\circ} 15$, Paris, $351 \mathrm{p}$.

Sawadogo L, Nygard R, Pallo F. 2002. Effects of livestock and prescribed fire on coppice growth after selective cutting of Sudanian savannah in Burkina Faso. Ann. For Sci., 59: 185-95.

Sokpon N, Biaou SH, Ouinsavi C, Hunhyet O. 2006. Bases techniques pour une gestion durable des forêts claires du Nord-Bénin: rotation, diamètre minimal d'exploitabilité et régénération. Bois For Trop, 287: 45-57.

Trochain J. 1970. Les territoires phytogéographiques de l'Afrique noire francophone d'après la trilogie - climat, flore et vegétation. CR. Séances Soc. Biogéogr., 395403: 139-157.

Yameogo TJ. 2006. Etude d'impact des aménagements antiérosifs sur la régénération ligneuse dans le massif forestier de Bougon. Province du Namentenga. Mémoire d'ingénieur IDR/UPB, 76. 